\section{DIE ERSTE SEITE}

\section{Dauer von Patientenkontakten}

\section{Nach knapp acht Minuten ist alles vorbei}

48 Sekunden in Bangladesch und 22,5 Minuten in Schweden - das markiert die Spanne der Sprechzeiten bei Allgemeinärzten aus 67 Ländern, die ein Team von der Universität Cambridge ermittelt hat. Im Sprechzimmer eines deutschen Hausarztes dauert ein Besuch im Schnitt sieben Minuten und 36 Sekunden. Die Forscher hatten 179 Studien analysiert, in denen insgesamt 28.570.712 Arztkonsultationen ausgewertet worden waren.

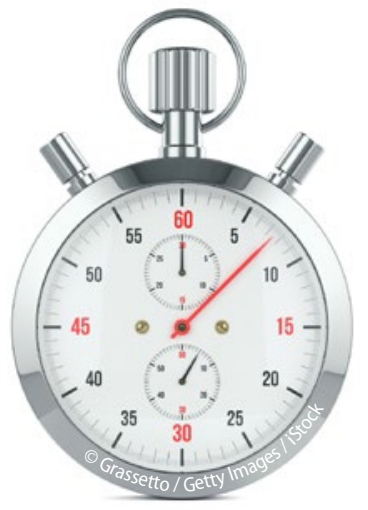

Die Autoren geben zu bedenken, kurze Konsultationszeiten könnten die Versorgung der Patienten beeinträchtigen, aber auch Ärzte belasten und Burn-out begünstigen. Eine Umfrage unter Hausärzten habe zudem gezeigt, dass mehr als ein Drittel von ihnen unzufrieden mit der Zeit sind, die sie für ihre Patienten aufbringen können.

- BMJ Open 2017; 7: e017902

https://doi.org/10.1136/bmjopen-2017-017902

\section{Hohe Ein-Jahres-Mortalität}

\section{„Hüftfraktur so zeitkritisch wie ein Schlaganfall!“}

Die Prognose älterer Patienten mit Hüftfraktur verschlechtert sich mit der Wartezeit bis zur Op. In einer Studie der Cleveland Clinic wurden Daten von 720 Patienten im Alter über 65 ausgewertet, bei denen eine Hüftfraktur operativ versorgt worden war. Zwischen Klinikaufnahme und Op.Beginn waren median 30 Stunden vergangen. 159 Patienten (22\%) verstarben im Jahr nach dem Eingriff. Das Risiko zu ster- ben stieg mit der Wartezeit. Nach Adjustierung für andere Risikofaktoren hatten Patienten, die erst nach über 60 Stunden in den OP kamen, ein fast dreimal so hohes Risiko, das Folgejahr nicht zu überleben, wie Patienten mit maximal 24-stündiger Verzögerung. Die Autoren fordern, Hüftfrakturen genauso dringlich zu behandeln wie Schlaganfälle oder Herzinfarkte.

- J Orthop Trauma 2017; online 14. Oktober

\section{Ursache für Knieverletzungen}

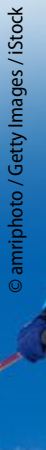

\section{Skibindung bei Frauen meist zu hart eingestellt}

Beim Skifahren verletzen sich Frauen doppelt so oft am Knie wie Männer. Sportwissenschaftler aus Innsbruck führen dies v. a. auf schlecht eingestellte Bindungen zurück. In ihrer Studie waren 60-80\% der Knieverletzungen im Hobbyskisport wegen nicht aufgegangener Bindungen beim Sturz passiert. Das Nichtauslösen der Bindung war bei Frauen um 20\% häufiger als bei Män-

nern. Den Experten zufolge krankt die ISONorm für Skibindungen daran, dass keine geschlechtsspezifischen Unterschiede berücksichtigt werden. So würde etwa eine Frau mit einer Körpergröße von $167 \mathrm{~cm}$ und einem Gewicht von $67 \mathrm{~kg}$ in denselben Bereich fallen wie ein $178 \mathrm{~cm}$ großer und 78 kg schwerer Mann. Durch einen entsprechenden Korrekturfaktor würde sich das Risiko für Knieverletzungen bei Skifahrerinnen wohl deutlich senken lassen.

- Br J Sports Med 2017; online 8. November; https://doi.org/10.1136/bjsports-2017-098572 